\title{
Teaching about Inequity: Shifts in Student Views about Diversity in Physics
}

\author{
Sierra R. Decker and Abigail R. Daane \\ Seattle Pacific University, Department of Physics \\ $33073^{\text {rd }}$ Ave. W., Seattle, WA, 98119, USA
}

\begin{abstract}
In an introductory university physics course, we designed and taught an equity unit focused on the effects of race and culture on the physics community. The goals of the unit are to identify areas of subjectivity in physics, analyze statistics about who participates in physics, justify the need for racial equity in physics, describe how obstacles such as implicit bias and stereotype threat can influence who participates in physics classes and professions, and feel empowered to take action. Across two years, many students' written reflections indicated that their views about equity in physics shifted as a result of participation in this unit. These students' responses fell into three categories, views that: changed, gained awareness, or solidified. We see these results as encouraging for instructors who wish to shift the physics community towards actively pursuing social justice by explicitly addressing equity in physics courses.
\end{abstract}

\section{INTRODUCTION}

Physics is often viewed as objective, uninfluenced by subjective lived experiences [1]. In reality, the study of physical phenomena is subjective, necessarily involving humans and their actions, perceptions, and interests [1,2]. Unfortunately, just as people of color and women are seriously underrepresented in physics [3, 4], so too are their actions, perceptions, and interests [5]. This inequitable representation is a disservice to those from marginalized groups who lack access to physics and is also detrimental to society, which does not benefit from additional brainpower. We suspect that, with few exceptions [6, 7], explicit discussions about inequity of representation do not occur often in physics classrooms. We take the perspective that people involved in physics should critically examine the ways in which privilege (e.g., whiteness) advantages some more than others [8]. Physicists and physics instructors can make a stronger commitment to discuss this issue with each other and students, working to increase access and inclusion for all marginalized groups [9]. In an effort to increase awareness of this problem, we implemented an equity unit into introductory physics classes. We hoped to see students better identify, articulate, and respond to the inequitable representation in physics. Our research question was: did this equity unit shift students' views on the importance of diversity in physics? To answer this question, we analyzed students' written reflections during the unit. In Sect. II, we give a brief overview of the equity unit, which we describe in detail elsewhere [10]. We then share research methods. In Sect. IV, we define three categories of student responses: their views that changed, gained awareness, or solidified. Finally, we provide potential mechanisms for this shift (Sect. IV) and areas of improvement for future work (Section V).

\section{OVERVIEW OF EQUITY UNIT}

Instruction and data collection occurred during the second quarter of introductory calculus-based physics courses at a small, religiously-affiliated, private university. The course meets four times a week for a total of about 6 hours per week. The classes integrate lab and lecture and are discussion-based, using round tables in groups of 4-6 students. Two sections of 30-45 students participated for two years, totaling 155 students. Approximately $20 \%$ of the students were female, although the university is $69 \%$ female. Table 1 shows the classroom racial demographics gathered from the university registration data for all four sections combined.

TABLE 1. Classroom Racial Demographics from University Registrar [11]

\begin{tabular}{lll}
\hline \hline Race & $\begin{array}{l}\text { Number } \\
\text { of Students }\end{array}$ & $\begin{array}{l}\text { Percentage } \\
\text { of Students }\end{array}$ \\
\hline Asian & 24 & $15 \%$ \\
Black or African American & 7 & $5 \%$ \\
Hawaiian Native or other & 1 & $1 \%$ \\
Pacific Islander & & \\
Hispanic of any race & 13 & $8 \%$ \\
Two or more races & 13 & $8 \%$ \\
White & 83 & $54 \%$ \\
Non-Resident Alien [11] & 14 & $9 \%$ \\
Total in four classes & $\mathbf{1 5 5}$ & $\mathbf{1 0 0 \%}$ \\
\hline \hline
\end{tabular}

Our research examined student responses during a fourday unit (3-5 instructional hours) designed to support student discussion and reflection on (primarily racial) issues of equity in physics [10]. The unit was originally designed to focus on a Supreme Court case regarding affirmative action, 
Fisher vs. UT-Austin (2016). Students began by discussing Justice Roberts' question, "What unique perspective does a minority [12] student bring to a physics class?" They grappled with whether or not the study of physics is influenced by race, culture, or diversity. Throughout the unit, students alternated between individual reflection and in-class discussion. Their readings included a letter that physicists wrote in response to Justice Roberts' question, a paper on stereotype threat, the Implicit Association Test, and statistics of who does physics [3-5,10]. In the last assignment, students reflected individually on what they could do to increase inclusivity in physics.

In Year 2, we asked students to define physics (and how it is subjective), instead of focusing on the court case. By supporting students in seeing how humans influence the study of physics, we could then argue for the benefits of having a representative sample of the population in the physics community. We added a follow-up reading that described various aspects of subjectivity in physics [2]. We then asked Justice Roberts' question, "What unique perspective does a minority bring to a physics class?" without emphasizing the court case. During the unit, we alternated among reflection, discussion, and readings. At the end of the unit, we added an opportunity in class for students to brainstorm ideas together about what they could do to make physics more equitable. This generated more ideas and supported students in moving from a space of hopelessness to action [13], something that they struggled with in Year 1 of the unit. The goals of the equity unit were for students to:

1) Identify areas of subjectivity in physics.

2) Analyze statistics about who participates in physics.

3) Justify the need for racial equity (inclusion and access) in physics.

4) Describe what and how obstacles such as implicit bias, stereotype threat, etc. can influence who participates in the physics field and classroom.

5) Feel empowered to create more equity in physics. Students were asked to reflect on their feelings and experiences that the unit produces and in this paper, we share student reflections that speak to goals 1,3 , and 4 .

\section{METHODS}

We collected written work from students as part of the regular class assignments. In effort to better understand the students' perceptions of the unit and gauge the productivity of the time spent on this topic in a physics class, we read student responses, searching for reflections that indicated a shift in views. All work was anonymized prior to any analysis (students' names were replaced with randomized numbers from 100 to 9999). Author 1 coded all open-ended responses (see Section IV for specific criteria). Author 2 independently coded $10 \%$ of the responses with an inter-rater reliability of $100 \%$. When students self-identified a shift in their views, their responses were grouped into emergent themes using key words or phrases that indicated a change [14]. Each response received a single code.

We were only able to place students in a category if they self-identified in writing that their views had shifted. During Year 1, we did not ask students to reflect on whether or not their ideas had shifted. Therefore, the statements we report here are unprompted and many students did not write about a change in their views. In Year 2, we asked, "Do you think your views changed based on our conversations and readings?" However, this question was an additional prompt under the broader question, "Why did we do this [unit] in intro physics?" and again, many students did not respond directly to the question. We report only those students who explicitly shared that the unit affected their views, even though others may have been impacted.

\section{STUDENT RESPONSES}

We found that 56 of 155 (36\%) students described their views about various aspects of equity and inclusion in physics as shifted, approximately one-third of each section. We categorized student responses into three emergent categories: views that A) changed, B) gained awareness, or C) solidified. We suggest possible mechanisms that supported those shifts.

\section{A. Changed}

Some students wrote explicitly that their views as changed. The majority of the 17 responses in this category came from Year 2. We marked the responses as aligned with this theme if they wrote about "changed" views (or a synonym such as altered) or had a response of the form "at first I thought $x$ but now I think $y$."

Students reported changes in their views in terms of the importance of diversity in general and the specific importance to the physics classroom. Several students described a perceived change about the importance of diversity in physics. For example,

Before, I thought that race wouldn't matter, but after talking to kids in the class and hearing about how they held back their ideas [because of their race], I now see the huge importance of having a diverse classroom. (395)

The latter student went on to describe a specific conversation in which their peers shared experiences with racism, helping this student to recognize the impact that racism and other challenges have had on them (goal 4). This student also described an increase in awareness for the importance of 
diversity in any classroom, whereas the former described diversity as important specifically in a physics class (goal 3).

Students also described an increased understanding of the nature of physics and its relationship to diversity (goal 1).

Over the course of this unit, my views have changed as a result of the reading and discussion. At the beginning, I was almost completely convinced that physics was only objective, and I had no idea about how diversity related to physics. Now, however, I have a better understanding about both of these topics. (7172)

These quotes represent shifts in views on the nature of physics, the importance of equity and its obstacles in physics.

\section{B. Gained Awareness}

Thirty students stated that they gained new information or new awareness during the equity unit. The responses in this category did not explicitly describe their views as changed, but instead that students learned something new, gained a new perspective, were previously unaware, or had their eyes opened. Here are two examples.

I very much appreciated this view on the topic, as it brought up a lot of points that I had not previously considered. Not being a minority student limits my ability to fully understand the struggles that minority students face daily, and it's not common that we have the opportunity to hear their concerns and hardships as minority students ... I hadn't before considered that it may take extra steps for the brilliant minds of minority students to feel able to pursue their education. (403)

Through the readings, I realized that diversity, in STEM and in physics, especially right now in our society, is very important ... I think it promoted a perspective I never really thought about. I think the powerful stories that I heard during small-group discussions made a big impact on what I should be looking out for and what to be aware of. I don't necessarily think I changed my views, but I think I was able to find new information and perspectives on certain aspects that I had not really bothered to look at. (1104)

These students wrote that they were impacted by the unit because it gave them the opportunity to learn more about specific challenges, such as stereotype threat, and experiences from students from marginalized groups that were new to them (goal 4).

\section{Solidified}

Nine students stated that the unit further solidified their views, giving them more information to support their current stance. Student responses in this category either stated that the unit helped the student ground, cement, or refine their views or gave them more data to support their views. One student stated that their views had not changed, and then reflected,

Wow! This was really good and helped me form a better stance ... I was unsure whether minorities in a physics classroom really made a difference because it's a hard science, but the letter had a great counterargument ... This week I've been able to see through the eyes of people where diversity does indeed make a remarkable difference in a physics class (and beyond!). (163)

Another student stated that this unit was beneficial for future career experiences.

Diversity is definitely important. Not only does it improve our communities with new ideas and perspectives, it is important for the people it creates opportunities for ... I don't know if I would say that my views changed, but I feel more strongly about them and I feel better prepared as I continue working toward a degree that will lead me to situations where this matters even more. (5228)

One student provided an analogy to describe their views.

I don't think my views have changed based on the conversations and readings, those two things just further cemented them into my brain. My best analogy of this is that the 'door' was already open, but after the conversation and articles the 'door' opening fully. (7068)

We categorized this response as solidified because of the explicit reference to cementing views. However, "the 'door' opening fully" analogy also supports a gained awareness. Students in both categories reported that this unit influenced them even though they had thought about the topic before in other contexts (goals $1 \& 3$ ). Many attributed their learning about equity to the physics context in which it occurred.

\section{Possible Mechanisms for Shifts}

Students cited various parts of the equity unit as supporting their shifts in views, most commonly: classroom discussions, the physicists' letter, and the readings in general. For example, many students reported that speaking with their peers about the problem of underrepresentation and hearing personal stories impacted them tremendously. One student wrote,

This conversation led me through a few different perspective changes. After the first discussion, I was led deeper into the conclusion that diversity was good because diverse students could bring new ideas to the 
table, new ways of thinking, new problem solving strategies and experiences. (597)

Students also stated that the physicists' letter influenced their views. These students wrote that hearing the view from physicists about how minorities deserve to be given the chance to be in physics opened their eyes to a new viewpoint on the situation. A student described their reaction,

[This letter] made me really reconsider my position on this issue of affirmative action due to [physicists'] arguments of institutional racism and trying to perfect the balance of standing of all races. (903)

The third main category was those who responded that the readings, such as the stereotype threat article, affected their views. They stated that these papers gave them information that helped influence their opinions. A student stated,

The readings are very insightful and made me think about this issue again. I learned how scientists think about the social issues and more about their beliefs. (8639)

\section{CONCLUSION \& FUTURE WORK}

Generally, students responded positively to the equity unit. One third of our students reported that the unit shifted their views about equity and inclusion in physics. Students described themselves as having A) changed their views (e.g., from not seeing racial diversity as important in physics to seeing it as important), B) gained awareness of specific challenges and issues of equity, or C) solidified ideas about how racial diversity contributes to more equity in physics.

The vast majority of students $(90 \%)$ wrote that their experience of the unit was positive, even though many did not write about an explicit shift in views. The remaining $10 \%$ of students explicitly voiced dissatisfaction with the unit, described elsewhere [15].

Seven responses included students who self-identified as people of color and wrote that their views had not changed.
However, they described the unit as important for others in the class. We share two examples here.

I am glad that this topic was talked about. As a minority student, I have often been the only black student in certain science classes. Often minority students don't take these classes and no one thinks twice about it... Thank you for bringing this topic to light. (159)

How I feel about this unit: I hope other students are able to remember this. Not just as an activity that points out prejudices still existent in the world, but as a word of encouragement. ... Being half Haole [Hawaiian], I've experienced prejudices from both sides. (6998)

These students wrote about how this unit can influence students for whom this is not a central issue but separated themselves from those students. In designing this unit, we were primarily concerned with increasing awareness of the lack of inclusion and equity. In the future, we need to improve learning for those students who already have experience regarding this topic, especially students of color.

These results provide evidence for positive shifts in students' views for goals 1, 3, and 4 in our courses. We encourage other physics instructors to discuss issues of equity and inclusion in their own classes, as is appropriate for their specific contexts. By increasing awareness of inequitable representation and support in contexts of physics learning, instructors may increase access and inclusion in the physics community. In the future, we plan to further investigate the impacts of this unit and adapt it to better support all students in developing a well-informed position about equity in physics.

\section{ACKNOWLEDGEMENTS}

Special thanks to Vashti Sawtelle, Moses Rifkin, Lane Seeley, Rachel E. Scherr, Laura Wood, and Amy D. Robertson for supporting the development of this work.

[9] Z. Hazari, G. Potvin, R. M. Lock., F. Lung, et al., Phys. Rev. Phys. Educ. Res. 9, (2013).

[10] A. R. Daane, S. Decker, and V. Sawtelle, Phys. Teach. 55(6), (2017).

[11] This data comes from the University registrar. E.g., "non-resident alien" records "international student" status and is included as a racial demographic.

[12] Any use of "minority" in this paper is a direct quote.

[13] B. D. Tatum, Teachers College Record 95(4), (1994).

[14] K. Krippendorff, Content Analysis: An Introduction to Its Methodology (Sage, CA, 2013), 3rd edn.

[15] A. R. Daane \& V. Sawtelle, AIP Conf. Proc., (2016). 